\title{
Fluid-Volume Assessment in the Investigation of Acute Heart Failure
}

\author{
Tertius Tuy $\cdot$ Martin Than
}

Published online: 14 March 2013

(c) Springer Science+Business Media New York 2013

\begin{abstract}
Heart failure is a syndrome whereby cardiac function is incapable of providing for the body's circulatory demands, resulting in hemodynamic and volumetric aberrations. Acute heart failure (AHF) is the condition where signs and symptoms of heart failure are present that require urgent intervention. In AHF impaired cardiac function progresses to hemodynamic congestion and eventually develops into a fluid overloaded state. These patients will typically present acutely for medical assistance, with signs and symptoms of fluid overload (dyspnea, paroxysmal nocturnal dyspnea, orthopnea, edema, etc.) and/or hypoperfusion (malaise, altered mental status, decreased urine output). In patients with AHF, volume assessment is a crucial step in the initial diagnosis.
\end{abstract}

Keywords Acute heart failure - Volume assessment . Echocardiography · Natriuretic peptide · Ultrasonography · Bioimpedance

\section{Introduction}

Although cardiac pressures are pivotal in understanding hemodynamics they often require admission to higher level facilities before they can be assessed accurately. Diagnosis of acute heart failure (AHF) may not be straightforward as patients may have other medical conditions which can confound the diagnostic picture (renal failure, liver disease,

T. Tuy $(\bowtie)$

Changi General Hospital, Singapore, Singapore

e-mail: tertius@gmail.com

M. Than

Christchurch Hospital, Christchurch, New Zealand obesity, infection etc.). Although the clinical picture may be unclear, in patients suspected of heart failure, it is important to identify the volume status during the initial examination [1]. ${ }^{1}$ Furthermore, volume assessment is pivotal in determining the mode and extent of volume reduction required.

There are multiple modes of volume assessment. Though some are more direct in their assessment than others, even indirect modalities, when used appropriately and in the proper context, are useful surrogates and give important information for clinical decision making. Considerations such as, time, costs, accuracy, radiation and availability effect the mode(s) of assessment selected. Although this chapter's primary aim is volume assessment, pressure and volume are intrinsically linked, thus attempts to talk about volume assessment without mentioning pressure assessment would be simplistic. Unfortunately, an in-depth discussion on hemodynamic monitoring exceeds the scope of this chapter and only current selected methods of volume assessment for AHF patients will be discussed.

\section{Pathophysiology}

Volume Overload in AHF

In AHF, myocardial impairment results in decreased diastolic or systolic function which is insufficient to maintain adequate perfusion to existing distal vascular resistance (the 'afterload'). This creates a backlog of fluid-volume and elevated ventricular filling pressures, which propagates into the lungs in left heart failure or systemically in right

\footnotetext{
1 The most up-to-date ACCF/AHA guidelines for diagnosis and management of heart failure.
} 
heart failure. In the kidney, the renal perfusion gradient diminishes as arterial pressures decreases and venous pressure increases from reduced cardiac output. Urine production is impaired and fluid is retained. Low renal perfusion can damage renal parenchyma which exacerbates kidney impairment. In patients with chronic heart failure, poor cardiac output leads to compensatory activation of the neurohormonal and renin-angiotensin-aldosterone system. As a result of high levels of aldosterone, patients may retain fluids. Lastly, there can be an inappropriate release of arginine vasopressin which up-regulates passive reabsorption of water in the kidney's collecting ducts [2].

\section{Central Venous Pressure and Cardiac Catheterization}

Central venous pressures (CVP) and cardiac catheterizations are used to optimize heart failure therapy, especially for cases of heart failure refractory to mainstay management. Pressure measurements are mainly done in settings with enhanced facilities by experienced physicians. Right heart catheterizations produce information about right atrial pressures and ventricular filling pressures, and SwanGanz catheterizations produce pulmonary capillary wedge pressures (PCWP) as a proxy for left atrial pressures. Delineating the etiology of the pressure rise can be complex; it can be unclear whether retention of fluids increases cardiac pressures or whether increased pressures cause a backlog of fluids. Furthermore, it is possible to have symptoms from pressure overload (e.g., j-receptor excitation), without evidence of vascular congestion. Although catheterization and CVP measurements are surrogate volume assessments, they are established methods of optimizing cardiac output and managing both preload and afterload reduction.

\section{Methods of Volume Assessment}

\section{The Gold Standard: Dilution Tracer Studies}

The gold standard for volume assessment is radioisotope dilution studies. After injecting a known volume and concentration of radioisotope tracer and allowing adequate time for equilibration, various volumes can be calculated (using different isotopes for the space in question). For calculation of total body water, heavy water (oxygen and hydrogen isotopes) are used. The lower the final concentration of radioisotope, the larger is the volume in question. Long equilibration times (4-6 h), costs of materials, cost of the measurement equipment, trained technicians, and exposure to radiation have made dilution studies clinically unsuitable for conditions with rapidly changing volumes such as AHF.

\section{Clinical Exam}

\section{History and Physical}

Depending on the experience of the physicians, eliciting the appropriate signs/symptoms of AHF may be difficult [3]. Patients with fluid overload from left heart failure may present with respiratory symptoms/signs such as dyspnea, orthopnea, paroxysmal nocturnal dyspnea, rales, audible third heart sound (S3). Patients with right heart failure may present with more systemic manifestations (swelling, jugular venous distension, abdominal jugular reflex, S3). A meta-analysis by Wang et al., examined the utility of clinical examination in predicting the presence of heart failure. Among the clinical symptoms (dyspnea on exertion, paroxysmal nocturnal dyspnea, orthopnea, edema), dyspnea on exertion had the highest sensitivity comparatively (see Table 1) [4]. Amongst clinical signs of volume overload, the abdominal jugular reflex, jugular venous distension and S3 had good specificity for AHF, but unfortunately lacked sensitivity (see Table 1) [4]. Rales and lower extremity edema were more sensitive and specific [4]. In general, when present, a positive clinical exam is useful; but a negative clinical exam cannot rule out the possibility of heart failure. Particularly in a loud and rushed environment (i.e., the ED) the signs and symptoms are not the most reliable method of assessment.

\section{Acoustic Cardiography}

Acoustic cardiography was developed to amplify and provide a readable signature of heart sounds intrinsically linked to the electrocardiogram. Since the S3 has a high specificity, acoustic cardiography has a potential in the evaluation of potential heart failure patients. In patients

Table 1 Clinical examination of fluid status in heart failure

\begin{tabular}{lll}
\hline & Sensitivity $(\%)$ & Specificity $(\%)$ \\
\hline Dyspnea on exertion & 84 & 34 \\
Paroxysmal nocturnal dyspnea & 41 & 84 \\
Orthopnea & 50 & 77 \\
Swelling & 51 & 76 \\
Abdominal jugular reflex & 24 & 96 \\
Jugular venous distension & 39 & 92 \\
Rales & 60 & 76 \\
Limb edema & 50 & 78 \\
S3 & 13 & 99 \\
\hline
\end{tabular}


who had a right heart catheterization, the use of acoustic cardiography predicted a PCWP $\geq 15 \mathrm{mmHg}$ ( $c$-stat 0.76 ) [5]. The Heart Failure and Audicor Technology for Rapid Diagnosis and Initial Treatment (HEARD-IT) trial investigated the use of acoustic cardiography S3 in 995 dyspneic patients and found that for acoustic cardiography the S3 had a sensitivity of $40.2 \%$ and a specificity of $88.5 \%$ [6]. Although it has low sensitivity, acoustic cardiography S3 was not found to change the diagnostic accuracy for AHF [6]. In the subset of patients with slightly to moderately raised brain natriuretic peptide (BNP) (between 100 and $499 \mathrm{pg} / \mathrm{mL}$ ), acoustic cardiography improved the accuracy of AHF diagnosis from 47 to $69 \%$ [7].

\section{Laboratory Tests}

\section{General}

The majority of blood results are poor indicators of volume status. Sodium and hemoglobin may be decreased from long standing activation of aldosterone leading to hemodilution but there are many other causes of hyponatremia and anemia to be considered [8], apart from a fluid overload state. Regardless, in the setting of AHF, their presence is more of an indicator of a poor prognosis [9-11] rather than severity of fluid overload. Likewise, urea and creatinine levels may be elevated in response to impaired kidney function from decreased perfusion (e.g., low cardiac output state). Depending on the clinical scenario, such elevations can be associated with a hypervolemia, hypovolemia, and euvolemia.

\section{Natriuretic Peptides}

Natriuretic peptides are elevated in the blood in response to cardiac stress. The BNP is the most commonly available. They serve not only as markers of heart strain but also as neurohormones promoting natriuresis and diuresis. They have high positive predictive value for AHF but do not entirely correlate with the volume status of a patient. Irrespective of volume status, natriuretic peptides may be elevated to varying degree in the elderly and in renal failure; while they may be inappropriately depressed in obese patients. A small pilot study by James et al., looked at blood volume and BNP values for ten CHF patients with pulmonary catheterization. The patients had an expanded blood volume approximately $30 \%$ more than baseline [12]. The pre-treatment and post-treatment BNP had poor correlation with blood volume (correlation factor $r=$ -0.127 and -0.126 , respectively) [12]. Another pilot study by Kazanegra et al., demonstrated that decreasing PCWP was correlated with dropping BNP levels $(r=0.79)$. The BNP and NT-proBNP have been investigated in other volume-overloaded states. A study of 31 dialysis patients without evidence of cardiac failure found that BNP and NT-proBNP values decreased with hemodialysis [13]. This drop did not correlate with the post hemodialysis fluid removal or weight decrease [13]. In another small study, Giglioli et al., assessed 15 patients with congestive heart failure undergoing slow continuous ultrafiltration. Fluid removal was associated with decreased pro-BNP levels and clinical improvement [14]. Overall, an elevated natriuretic peptide level is of concern and should be collaborated with the clinical context in determining whether fluid retention is present.

\section{Imaging}

\section{Chest Radiography}

The chest radiograph is a common and convenient method to assess for accumulation of fluid within the lungs. It is cheap and readily available in almost all institutions. Patients with certain classically radiographic signs (pulmonary vascular redistribution, Kerley B lines, alveolar edema, pleural effusions, and bilateral infiltrates) [15] are likely to be retaining fluids in the lungs and, with an appropriate history, they are highly specific for AHF. In fact, for the dyspneic ED patient, while increased distribution and flow to the superior pulmonary vessels (cephalization), interstitial edema and alveolar edema had poor sensitivity $(41,27$, and $6 \%$, respectively) for $\mathrm{AHF}$, it was highly specific $(96,98$, and $99 \%$, respectively) (see Table 2) [16]. When found on chest radiography, cardiomegaly does not in itself imply retention of fluids, yet it indicates history of heart failure, and provides a possible etiology for the fluid overloaded state. The Acute Decompensated Heart Failure National Registry (ADHERE) demonstrated that about $19 \%$ of AHF patients presenting to the ED will have an apparently normal chest radiograph, and up to $10 \%$ of non-AHF patients admitted from the ED had a positive chest radiograph [17•].

Vascular pedicle width (VPW) is the horizontal distance from the exit point of the left subclavian artery from the aortic arch to the intersection of the SVC and the right mainstem bronchus [18]. Although the number of studies of VPW and fluid overload is limited and numerical normal values have not been fully established, a meta-analysis by Wang et al. [18] found that for volume overloaded patients

Table 2 Radiographic signs of heart failure on chest X-ray

\begin{tabular}{lll}
\hline & Sensitivity $(\%)$ & Specificity $(\%)$ \\
\hline Cephalization & 41 & 96 \\
Interstitial edema & 27 & 98 \\
Alveolar edema & 6 & 99 \\
\hline
\end{tabular}


the VPW for erect and supine patients was 52.2 and $71.1 \mathrm{~mm}$ respectively, and overall the VPW was strongly correlated with volume overload $(r=0.81, p=0.80)$. Studler et al. [19], however, found that the diagnostic accuracy of supine VPW's in isolation for determining heart failure was moderate at best ( $c$-stat 0.461).

\section{Echocardiography}

Two-dimensional echocardiography is useful in determining the cardiac function as well as looking at cardiac anatomy. Although it displays a two dimensional image of the heart, it can assess for chamber dilation and can suggest the presence of cardiac fluid overload. Several algorithms have been developed to approximate fluid volume. The three dimensional echocardiogram largely avoids the use of algorithms and has been found to be more precise in the evaluation of end diastolic and end-systolic volumes as well as ejection fraction approximation [20]. Three dimensional echocardiograms of the right atrial volume index correlate with right atrial pressures $(r=0.51, p<0.001)$ and high right atrial volume index $\left(\geq 35 \mathrm{~mL} / \mathrm{m}^{2}\right)$ is predictive of right atrial pressures $>10 \mathrm{mmHg}$ [21]. Despite being useful for evaluation of AHF, echocardiography still underestimates ventricular volumes compared to cardiac MRI [22]. Serial echocardiography can provide insight into the response to preload reduction through improvement of cardiac function.

\section{Ultrasonography}

Aside from echocardiography, ultrasonography has been implemented to assess fluid status based on imaging of the lung fields and the inferior vena cava (IVC).

Ultrasound Lung Comets Extravascular lung volume may be assessed by ultrasonography of the anterior chest between the second and fifth intercostal space looking for signs of interlobular septal fluid called ultrasound lung comets (ULCs) [23]. This technique is easily learned (learning curve of $<10$ examinations) and quick to perform ( $<3 \mathrm{~min}$ ) [23]. In post cardiac surgery patients, presence of ULCs correlated with an increase in wedge pressure ( $r=0.48, p=0.01$ ) [24] as well as presence of radiologic evidence of fluid overload $(r=0.60, p=0.0001)$ [24]. Additionally, for dyspneic patients, the number of ULC was highly correlated to NT-proBNP $(r=0.69, p<$ $0.0001)$ and was good at predicting cardiogenic dyspnea ( $c$-stat $0.893, p=0.001$ ) [25]. The number of ULCs was associated with the New York Heart Association class $(\mathrm{OR}=2.1, \mathrm{CI}=1.4-2.9)$ and the degree of diastolic dysfunction $(\mathrm{OR}=2.438, \mathrm{CI}=1.418-4.190) \quad$ [26]. A small study of HF patients with intracardiac defibrillator, by Maines et al. [27] showed that, in HF patients with ICD, that having $\geq 5$ ULCs during regular follow-up had a sensitivity of $83 \%$ and positive predictive value $91 \%$ for clinical HF deterioration. In addition, having a higher degree of extravascular lung water as assessed by ULCs connotes a poor prognosis. Patients with no ULCs had a three-fold improvement in a 16 month event-free survival compared to patients with many ( $>30$ ) ULCs ( 70 vs. $19 \%$, $p=0.0007$ ) [28]. The utility of ULCs is still being investigated, but currently it has not gained clinical popularity.

Inferior Vena Cava Measurements Another method of assessing fluid status is by ultrasound of the IVC. The IVC is in direct communication with the right atrium through which pressure and volume can propagate. In volumeoverloaded states the diameter of the IVC expands while the collapsibility diminishes. Measurement is taken within $2.0 \mathrm{~cm}$ of the IVC and right atrial junction [29]. The maximum and minimum diameter is defined as the largest and smallest diameter of the IVC during a passive respiratory cycle. In patients with fluid overload (e.g., CHF) variation with respiration is diminished or absent [30]. In a study of patients referred for a right heart catheterization, a hand-carried ultrasonography was performed looking at the IVC diameter. It found that a maximum IVC diameter of $2.1 \mathrm{~cm}$ was predictive of having a PCWP $\geq 15 \mathrm{mmHg}$ (c-stat 0.89) [31]. Moreover, among 102 patients undergoing right heart catheterization, the IVC minimum and maximum diameter during passive respiration correlated with right atrial pressures $(r=0.50-0.60)$ [29]. Additionally, an IVC maximum diameter of $2.0 \mathrm{~cm}$ and a minimum diameter of $1.5 \mathrm{~cm}$ could predict a right atrial pressure of $\geq 10 \mathrm{mmHg}$ ( $c$-stat 0.76 and 0.88 , respectively) [29]. Thus, a maximum IVC diameter of $2.0 \mathrm{~cm}$ had a sensitivity and specificity of 73 and $85 \%$ while a minimum IVC diameter of $1.5 \mathrm{~cm}$ had a sensitivity and specificity of 91 and $79 \%$ [29]. Lastly, patients admitted for HF with larger IVC size had an increased 30-day readmission rate [32].

The IVC collapsibility index (IVC-CI) is defined as the difference between the maximum and the minimum IVC diameter all divided by the maximum IVC diameter. It is thought to decrease with increasing vascular congestion, and in patients, as mean CVP decreases, the IVC-CI increases $(p=0.023)$ [33]. In patients with suspected right heart failure, a low IVC-CI $(0.22)$ had a sensitivity and specificity for right heart failure of 78 and $98 \%$, respectively [34]. Also, the IVC-CI has been used to follow acute volume changes. Patients undergoing ultrafiltration with a total ultrafiltrate production of $5,780 \mathrm{~mL}$, had a significantly increased IVC-CI $(p<0.001)$, while there was no change in mean arterial pressures, heart rate, or the IVC diameter [35]. 


\section{Bioimpedance}

Bioimpedance uses a small electrical current (of various frequencies) to measure the inherent resistance and reactance between placed electrodes. Higher fluid content results in lower resistance and reactance values. There are a wide variety of bioimpedance devices and multiple modes of measurements. Impedance cardiography interprets this principle in order to calculate the hemodynamics (systemic vascular resistance, cardiac output, etc.). Bioimpedance analysis (BIA) indirectly measures fluid content between electrodes. It can be used to calculate total body water or water within a body segment. There are bioimpedance devices that are extracorporeal and those that are implanted along with intracardiac devices.

\section{Bioimpedance Analysis, Bioimpedance Vector Analysis, and Bioimpedance Spectroscopy}

The BIA has been well established to be strongly correlated with total body water ( $r=0.996$ ) as assessed by deuterium labeled water [36]. It utilizes a non-invasive technique which places external electrodes on the hands and/or feet in order to attain the most accurate results, the position of the patient is supine with the hands $30^{\circ}$ away from the body and feet $45^{\circ}$ apart. This is limiting in cases where patients are not able to lie flat (orthopnea, severe back pain) or where bed sizes may be limited (small beds, obese patients). In addition, single frequency bioimpedance is not capable of differentiating extracellular from intracellular fluids. Whole body BIA has been used in differentiating dyspnea in AHF and non-AHF patients. In 292 dyspneic patients presenting to the ED, patients with AHF were found to have lower whole body (402.3 vs. $513.2 \Omega$ ) and segmental (35.5 vs. $66.4 \Omega$ ) resistance [37]. Both segmental and whole body BIA had a significant correlation with BNP values [37] and patients undergoing diuretic therapy for heart failure had a significant increase in resistance and reactance post therapy $[38,39]$. Finally, a study by Paterna et al., found that BIA could be used to monitor resolution of fluid overload in heart failure patients [39].

Similar to BIA, bioimpedance vector analysis (BIVA) has also been investigated as volume assessment tool in AHF. The BIVA plots height standardized reactance and resistance values to determine volume status based from previous population data. In addition, BIVA is capable of giving a calculated hydration state $(\%)$. In patients with AHF undergoing $72 \mathrm{~h}$ of diuretic therapy, BIVA's hydration status was successful in monitoring diuresis and helped identify patients who attained their "dry weight" [40]. In patients who had an average hydration value $>80.5 \%$ there was an increase likelihood for death or rehospitalization for cardiogenic event ( $+\operatorname{LR} 4.6, p=0.04$ ) [40]. Another variation to BIA is bioimpedance spectroscopy (BIS), which uses 50 frequencies from 5 to $1,000 \mathrm{kHz}$ to calculate impedance and hydration values. The advantage is that in addition to total body water, the extracellular and intracellular components can be measured. The BIS has been predominantly used for hemodialysis in attaining dry weight [41], and, at present, there are few if any studies that investigates the utility of BIS in AHF.

\section{Intrathoracic Impedance}

Presently certain intracardiac devices have used bioimpedance to detect accumulation of lung and/or thoracic fluids. In this manner the goal of intrathoracic impedance is to identify patients at risk for decompensation before an overt episode of AHF. By monitoring the intrathoracic impedance values (alerts set to a predefined threshold), patients with congestive heart failure at risk for decompensating within the next 30 days could be identified [42]. In SENSE-HF, bioimpedance predicted hospitalization for heart failure with a sensitivity of $42.1 \%$ [43]. A study by Catanzariti et al. [44] found if therapy was adjusted in view of the alerts, heart failure hospitalizations could be reduced in patients with alerts compared to without alerts ( 7 vs. $20 \%, p<0.001$ ). Other studies have established that intrathoracic impedance and audible alerts actually increased hospitalization (hazard ratio $1.79, p=0.022$ ) compared to the controls [45]. This finding might be from improper hospitalization from overreaction to the alert itself. Further studies are needed to expound on the rationale for the difference between these studies.

\section{Summary}

- Although certain clinical exam symptoms and signs are predictive of fluid overload, they are not always present and their sensitivities are low.

- Acoustic Cardiography of S3 can improve the pickup rate and may increase the sensitivity for the detection of the S3 heart sound.

- Chest radiography looking for signs of fluid overload remains part of the in hospital standard of care, yet signs are not always present and may lag behind clinical symptoms by several hours.

- Natriuretic peptides such as BNP are an indicator of cardiac stress and tend to be elevated in fluid overload states, but do not completely correlate with fluid-volume status. They are a good indicator of poor prognosis for the patient.

- Ultrasound technology is used to approximate cardiac chamber volume and to assess volume status through 
detecting thoracic fluid in the lungs (lung comets) or measuring IVC diameter.

- Bioimpedance technology is a promising non-invasive means of volume assessment, which may be implemented intra and extrathoracically. More research is required.

\section{Conclusion}

Fluid-volume assessment is a crucial part of the clinical evaluation and management of AHF. Multiple methods of volume assessment exist that are becoming increasingly accurate. Minimally or non-invasive forms of volume assessment are emerging that are readily available in several institutions around the world. These include natriuretic peptides, imaging studies, and bioimpedance. Regardless of the technology, these results must be incorporated into the overall clinical picture.

Disclosure No potential conflicts of interest relevant to this article were reported.

\section{References}

Papers of particular interest, published recently, have been highlighted as:

- Of importance

1. Jessup M, Abraham WT, Casey DE, Feldman AM, Francis GS, Ganiats TG, et al. 2009 Focused update: ACCF/AHA Guidelines for the Diagnosis and Management of Heart Failure in Adults: a report of the American College of Cardiology Foundation/ American Heart Association Task Force on Practice Guidelines: developed in collaboration with the International Society for Heart and Lung Transplantation. Circulation. 2009;119(14):1977-2016.

2. Cotter G, Metra M, Milo-Cotter O, Dittrich HC, Gheorghiade M. Fluid overload in acute heart failure-re-distribution and other mechanisms beyond fluid accumulation. Eur J Heart Fail. 2008;10(2):165-9.

3. Stevenson LW, Perloff JK. The limited reliability of physical signs for estimating hemodynamics in chronic heart failure. JAMA. 1989;261(6):884-8.

4. Wang CS, FitzGerald JM, Schulzer M, Mak E, Ayas NT. Does this dyspneic patient in the emergency department have congestive heart failure? JAMA. 2005;294(15):1944-56.

5. Collins SP, Kontos MC, Michaels AD, Zuber M, Kipfer P, Attenhofer Jost C, et al. Utility of a bedside acoustic cardiographic model to predict elevated left ventricular filling pressure. Emerg Med J. 2010;27(9):677-82.

6. Collins SP, Peacock WF, Lindsell CJ, Clopton P, Diercks DB, Hiestand B, et al. S3 detection as a diagnostic and prognostic aid in emergency department patients with acute dyspnea. Ann Emerg Med. 2009;53(6):748-57.

7. Maisel AS, Peacock WF, Shah KS, Clopton P, Diercks D, Hiestand B, et al. Acoustic cardiography S3 detection use in problematic subgroups and B-type natriuretic peptide "gray zone": secondary results from the heart failure and audicor technology for rapid diagnosis and initial treatment multinational investigation. Am J Emerg Med. 2011;29(8):924-31.

8. Nanas JN, Matsouka C, Karageorgopoulos D, Leonti A, Tsolakis E, Drakos SG, et al. Etiology of anemia in patients with advanced heart failure. J Am Coll Cardiol. 2006;48(12):2485-9.

9. Androne AS, Katz SD, Lund L, LaManca J, Hudaihed A, Hryniewicz $\mathrm{K}$, et al. Hemodilution is common in patients with advanced heart failure. Circulation. 2003;107(2):226-9.

10. Felker GM, Gattis WA, Leimberger JD, Adams KF, Cuffe MS, Gheorghiade M, et al. Usefulness of anemia as a predictor of death and rehospitalization in patients with decompensated heart failure. Am J Cardiol. 2003;92(5):625-8.

11. Baldasseroni S, Urso R, Orso F, Bianchini BP, Carbonieri E, Ciro A, et al. Relation between serum sodium levels and prognosis in outpatients with chronic heart failure: neutral effect of treatment with beta-blockers and angiotensin-converting enzyme inhibitors: data from the Italian Network on Congestive Heart Failure (IN-CHF database). J Cardiovasc Med (Hagerstown). 2011;12(10):723-31.

12. James KB, Troughton RW, Feldschuh J, Soltis D, Thomas D, Fouad-Tarazi F. Blood volume and brain natriuretic peptide in congestive heart failure: a pilot study. Am Heart J. 2005;150(5):984.

13. Bargnoux AS, Klouche K, Fareh J, Barazer I, Villard-Saussine S, Dupuy AM, et al. Prohormone brain natriuretic peptide (proB$\mathrm{NP}$ ), BNP and N-terminal-proBNP circulating levels in chronic hemodialysis patients. Correlation with ventricular function, fluid removal and effect of hemodiafiltration. Clin Chem Lab Med. 2008;46(7):1019-24.

14. Giglioli C, Landi D, Gensini GF, Valente S, Cecchi E, Scolletta $\mathrm{S}$, et al. Cardiac efficiency improvement after slow continuous ultrafiltration is assessed by beat-to-beat minimally invasive monitoring in congestive heart failure patients: a preliminary report. Blood Purif. 2010;29(1):44-51.

15. Chait A, Cohen HE, Meltzer LE, VanDurme JP. The bedside chest radiograph in the evaluation of incipient heart failure. Radiology. 1972;105(3):563-6.

16. Knudsen CW, Omland T, Clopton P, Westheim A, Abraham WT, Storrow AB, et al. Diagnostic value of B-type natriuretic peptide and chest radiographic findings in patients with acute dyspnea. Am J Med. 2004;116(6):363-8.

17. • Collins SP, Lindsell CJ, Storrow AB, Abraham WT. Prevalence of negative chest radiography results in the emergency department patient with decompensated heart failure. Ann Emerg Med. 2006;47(1):13-8. The ADHERE Registry, with over 100,000 patients enrolled, is one of the largest databases in the world on acute heart failure.

18. Wang H, Shi R, Mahler S, Gaspard J, Gorchynski J, D'Etienne J, et al. Vascular pedicle width on chest radiograph as a measure of volume overload: meta-analysis. West J Emerg Med. 2011;12(4): 426-32.

19. Studler U, Kretzschmar M, Christ M, Breidthardt T, Noveanu M, Schoetzau A, et al. Accuracy of chest radiographs in the emergency diagnosis of heart failure. Eur Radiol. 2008;18(8):1644-52.

20. Dorosz JL, Lezotte DC, Weitzenkamp DA, Allen LA, Salcedo EE Performance of 3-dimensional echocardiography in measuring left ventricular volumes and ejection fraction: a systematic review and meta-analysis. J Am Coll Cardiol. 2012;59(20):1799-808.

21. Patel AR, Alsheikh-Ali AA, Mukherjee J, Evangelista A, Quraini D, Ordway LJ, et al. 3D echocardiography to evaluate right atrial pressure in acutely decompensated heart failure correlation with invasive hemodynamics. JACC Cardiovasc Imaging. 2011;4(9):938-45.

22. Moceri P, Doyen D, Bertora D, Cerboni P, Ferrari E, Gibelin P. Real time three-dimensional echocardiographic assessment of left ventricular function in heart failure patients: underestimation of left ventricular volume increases with the degree of dilatation. Echocardiography. 2012;29:970-7. 
23. Picano E, Frassi F, Agricola E, Gligorova S, Gargani L, Mottola G. Ultrasound lung comets: a clinically useful sign of extravascular lung water. J Am Soc Echocardiogr. 2006;19(3):356-63.

24. Agricola E, Bove T, Oppizzi M, Marino G, Zangrillo A, Margonato A, et al. "Ultrasound comet-tail images": a marker of pulmonary edema: a comparative study with wedge pressure and extravascular lung water. Chest. 2005;127(5):1690-5.

25. Gargani L, Frassi F, Soldati G, Tesorio P, Gheorghiade M, Picano E. Ultrasound lung comets for the differential diagnosis of acute cardiogenic dyspnoea: a comparison with natriuretic peptides. Eur J Heart Fail. 2008;10(1):70-7.

26. Frassi F, Gargani L, Gligorova S, Ciampi Q, Mottola G, Picano E. Clinical and echocardiographic determinants of ultrasound lung comets. Eur J Echocardiogr. 2007;8(6):474-9.

27. Maines M, Catanzariti D, Angheben C, Valsecchi S, Comisso J, Vergara G. Intrathoracic impedance and ultrasound lung comets in heart failure deterioration monitoring. Pacing Clin Electrophysiol. 2011;34(8):968-74.

28. Frassi F, Gargani L, Tesorio P, Raciti M, Mottola G, Picano E. Prognostic value of extravascular lung water assessed with ultrasound lung comets by chest sonography in patients with dyspnea and/or chest pain. J Card Fail. 2007;13(10):830-5.

29. Brennan JM, Blair JE, Goonewardena S, Ronan A, Shah D, Vasaiwala S, et al. Reappraisal of the use of inferior vena cava for estimating right atrial pressure. J Am Soc Echocardiogr. 2007; 20(7):857-61.

30. Blehar DJ, Dickman E, Gaspari R. Identification of congestive heart failure via respiratory variation of inferior vena cava diameter. Am J Emerg Med. 2009;27(1):71-5.

31. Goonewardena SN, Blair JE, Manuchehry A, Brennan JM, Keller $\mathrm{M}$, Reeves R, et al. Use of hand carried ultrasound, B-type natriuretic peptide, and clinical assessment in identifying abnormal left ventricular filling pressures in patients referred for right heart catheterization. J Card Fail. 2010;16(1):69-75.

32. Goonewardena SN, Gemignani A, Ronan A, Vasaiwala S, Blair J, Brennan JM, et al. Comparison of hand-carried ultrasound assessment of the inferior vena cava and $\mathrm{N}$-terminal pro-brain natriuretic peptide for predicting readmission after hospitalization for acute decompensated heart failure. JACC Cardiovasc Imaging. 2008;1(5):595-601.

33. Stawicki SP, Braslow BM, Panebianco NL, Kirkpatrick JN, Gracias VH, Hayden GE, et al. Intensivist use of hand-carried ultrasonography to measure IVC collapsibility in estimating intravascular volume status: correlations with CVP. J Am Coll Surg. 2009;209(1):55-61.
34. Goei R, Ronnen HR, Kessels AH, Kragten JA. Right heart failure: diagnosis via ultrasonography of the inferior vena cava and hepatic veins. Rofo. 1997;166(1):36-9.

35. Guiotto G, Masarone M, Paladino F, Ruggiero E, Scott S, Verde $\mathrm{S}$, et al. Inferior vena cava collapsibility to guide fluid removal in slow continuous ultrafiltration: a pilot study. Intensive Care Med. 2010;36(4):692-6.

36. Kushner RF, Schoeller DA, Fjeld CR, Danford L. Is the impedance index (ht $2 / \mathrm{R}$ ) significant in predicting total body water? Am J Clin Nutr. 1992;56(5):835-9.

37. Parrinello G, Paterna S, Di Pasquale P, Torres D, Fatta A, Mezzero M, et al. The usefulness of bioelectrical impedance analysis in differentiating dyspnea due to decompensated heart failure. J Cardiac Fail. 2008;14(8):676-86.

38. Coodley EL, Segal JL, Smith DH, Neutel JM. Bioelectrical impedance analysis as an assessment of diuresis in congestive heart failure. Ann Pharmacother. 1995;29(11):1091-5.

39. Paterna S, Di Pasquale P, Parrinello G, Fornaciari E, Di Gaudio F, Fasullo S, et al. Changes in brain natriuretic peptide levels and bioelectrical impedance measurements after treatment with highdose furosemide and hypertonic saline solution versus high-dose furosemide alone in refractory congestive heart failure: a doubleblind study. J Am Coll Cardiol. 2005;45(12):1997-2003.

40. Di Somma S, De Berardinis B, Bongiovanni C, Marino R, Ferri $\mathrm{E}$, Alfei B. Use of BNP and bioimpedance to drive therapy in heart failure patients. Congest Heart Fail. 2010;16:S56-61.

41. Chazot C, Wabel P, Chamney P, Moissl U, Wieskotten S, Wizemann $\mathrm{V}$. Importance of normohydration for the long-term survival of haemodialysis patients. Nephrol Dial Transplant. 2012;27:2404-10.

42. Sarkar S, Hettrick DA, Koehler J, Rogers T, Grinberg Y, Yu CM, et al. Improved algorithm to detect fluid accumulation via intrathoracic impedance monitoring in heart failure patients with implantable devices. J Card Fail. 2011;17(7):569-76.

43. Conraads VM, Tavazzi L, Santini M, Oliva F, Gerritse B, Yu CM, et al. Sensitivity and positive predictive value of implantable intrathoracic impedance monitoring as a predictor of heart failure hospitalizations: the SENSE-HF trial. Eur Heart J. 2011;32(18):2266-73.

44. Catanzariti D, Lunati M, Landolina M, Zanotto G, Lonardi G, Iacopino $\mathrm{S}$, et al. Monitoring intrathoracic impedance with an implantable defibrillator reduces hospitalizations in patients with heart failure. Pacing Clin Electrophysiol. 2009;32(3):363-70.

45. van Veldhuisen DJ, Braunschweig F, Conraads V, Ford I, Cowie $\mathrm{MR}$, Jondeau $\mathrm{G}$, et al. Intrathoracic impedance monitoring, audible patient alerts, and outcome in patients with heart failure. Circulation. 2011;124(16):1719-26. 